\title{
A United Kingdom Pilot Study of Kinetic Shift Therapy and Mindfulness Based Therapy for Clients Living with Intellectual Disabilities and Enduring Mental Health Problems
}

\author{
Jo Collins-McGroarty ${ }^{1}$, Elizabeth Faulkes-Sharrock ${ }^{2}$, and Raquel Wilkes ${ }^{3, *}$ \\ ${ }^{1}$ NHS England Leeds, Tavistock and Portman NHS Trust, University of Essex, England \\ ${ }^{2}$ The Berne Institute, Nottingham, England \\ ${ }^{3} \mathrm{NHS}$ Leeds Foundation NHS Trust, Tutor Leeds Beckett's University, England
}

*Corresponding author: Raquel Wilkes, NHS Leeds Foundation NHS Trust, Tutor Leeds Beckett's University, England, E-mail: racquel.wilkes@nhs.net

Received: 18 Aug, 2020 | Accepted: 10 Nov, 2020 | Published: 16 Nov, 2020

Citation: Collins-McGroarty J, Faulkes-Sharrock E, Wilkes R (2020) A United Kingdom Pilot Study of Kinetic Shift Therapy and Mindfulness Based Therapy for Clients Living with Intellectual Disabilities and Enduring Mental Health Problems. J Psychiatry Ment Health 6(1): dx.doi. org/10.16966/2474-7769.137

Copyright: (c) 2020 Collins-McGroarty J, et al. This is an open-access article distributed under the terms of the Creative Commons Attribution License, which permits unrestricted use, distribution, and reproduction in any medium, provided the original author and source are credited.

\begin{abstract}
Objective: Clients with moderate intellectual disabilities are reported to struggle with ongoing mental health problems, especially around emotional and behavioral issues around sensitive topics like their sexuality, which is not always easy to communicate through traditional talking therapies for this client group, therefore a group of individuals were selected to determine the impact of a newer energy psychotherapy technique called Kinetic Shift Therapy (KS) alongside Mindfulness based therapy (MBT) which is utilized more, however with limited published research to determine the positive impact on the symptoms and behaviors that affect this client group.
\end{abstract}

Method: A quasi experimental method was chosen with a one test pretest/posttest design was used on 12 male clients attending a local charity support group for those living with intellectual disabilities who have been medically diagnosed with intellectual disabilities in accordance with the DSM-5. Self-assessment tools were utilized for the client and their regular care's which examined their emotional and behavioral issues.

Results: Variance was repeated and significant impact of the KS and Mindfulness combined therapy. The effect of variance was above average and an improvement was shown on self-harm, depression, aggression and anxiety symptoms.

Conclusion: This pilot study suggests that a newer energy psychotherapy intervention (KS) alongside (MBT) can reduce the symptoms and behaviors of this population to improve their overall well-being. It is recommended that further trials commence to determine the efficacy of these therapies on this population over a longer period of time.

Keywords: Mindfulness Interventions/Therapy; Intellectual Disabilities; Anxiety; Depression; Self-Harm; Kinetic Shift; Aggression; Sexuality; Energy Psychology

Abbreviation: AMM: Adolescent Mindfulness Measure; BAS: Behavioral Assessment System; KS: Kinetic Shift; MBT: Mindfulness Based Therapy; NICE: National Institute of Clinical Excellence; SD: Standard Deviation; ANOVA: Analysis of Variance

\section{Background of the Pilot Study}

Intellectual disability is a lifelong condition, resulting in reduced intellectual ability [1]. Some people may have a mild intellectual disability, while others may have profound issues. Intellectual disability limits people from intellectual functioning and impacts everyday tasks, for example accessing health care, or recognizing and responding to, or expressing changes in health. There are several therapeutic treatments used when helping those living with an intellectual disability, including carrying out psychotherapy, the chosen method for this research test was a newer energy based imagery psychotherapy called Kinetic Shift practiced by psychotherapists around the globe and developed in the UK to help individuals who may struggle with expressing their emotions during therapy. This pilot study seeks to determine the effectiveness of Kinetic Shift, alongside a Mindfulness therapy approach for those living with an intellectual disability who struggle with mental health issues, emotional regulation and self-harming behavior surrounding their sexuality, [1]. People with learning disabilities can be lesbian, gay, bisexual and transgender just like anyone else, [2], these groups are at risk of been bullied or harassed due to their learning problem and issues around their sexuality which impact their mental well-being. Energy Psychology methods like Kinetic Shift is a "mind and body 
approach" states therapist Karl Smith who developed the Kinetic Shift approach [3], and combines exposure therapy-a widely researched technique which has been established for a long time [4]. Early developments of energy psychology such as the increasingly popular Emotional Freedom Technique also known as "EFT or tapping", which also releases negative thoughts, feelings and emotions to promote mental well-being were developed by psychologists and therapists such as Fred Gallo, Roger Callaghan and David Feinstein. EFT is another form of exposure therapy and cognitive re-framing with the option of not having to discuss the actual mental health problem or root cause in great depth causing further distress [4].

The exposure/energy technique used in this study involved imagining a difficult memory/event, behavior, thought or feeling for the client, with the help of a qualified practitioner helping the client re process/re-frame and release the difficult feelings or symptoms/ behavior through the Kinetic Shift protocol to improve their wellbeing. The benefit to this client group is that they do not have to discuss their problems in depth or narrate them and can tell their story in shapes, colors and what it feels like in the body. The theory behind this approach is to integrate the mind and body and to reprocess events in a safe environment. Smith asserts that the person becomes engaged in their feelings, thoughts and behaviors and these are then targets for change. This type of therapy is highly beneficial states Smith [3], for client's with an intellectual disability to aid their rehabilitation towards a better mental health. The principle behind utilizing Kinetic Shift alongside Mindfulness is due to the ongoing positive testimonials and anecdotal reports from client's around the Globe with multiple issuessuch as learning issues, trauma/PTSD, phobias, eating problems, behavioral issues, addictions and anxiety disorders, which have all been reported with success however further randomized research studies are still needed. Mindfulness therapy is perhaps the most frequently reported approach for those with intellectual disabilities; however according to the National Institute of Clinical Excellence in the UK research remains limited, which the authors of this study concluded [5].

According to the World Health Organization (WHO), in 2011, 15\% of the world's population lives with a disability, $4 \%$ have a significant mental health disability like an intellectual disability, and this is set to rise due to an increase in the global population. WHO states in their mental health reports that many of the world's population do not have access to timely effective psychological therapy due to lack of training in complex areas and communication barriers which affect largely those with intellectual disabilities. The authors of this study assert the need for a more flexible and integrated/holistic approach to mental health care especially with psychological therapies when dealing with individuals with complex needs such as intellectual disabilities.

People with intellectual disability often struggle with learning, understanding, expressing their needs, discussing emotions and at times expressing issues around sex and their sexuality. People with intellectual disabilities have a difficult time identifying their sexual rights in practice [6]. These populations are more vulnerable to sexual abuse, and lack the skills and knowledge of their sexuality [6], they experience resistance from their environment when practicing their sexuality. People with intellectual disabilities struggle with understanding instructions, doing maths, reading and writing. Individuals find it difficult to understand when studying tests and staying focused. Those with intellectual disabilities are often unemployed, socially lonely, and stigmatized and bullied, [6]. Those living with learning needs have greater health and social care needs, [7] additional support and adjustments may be required.
Health care professionals and therapist's require an awareness of the complex individual needs of this population; therapy needs to include the use of images, symbols, gestures and objects of reference to allow expression of painful emotions. Family and carers are valuable however the authors assert they have found at times this to be a barrier to therapy when personal information is disclosed, wherever possible the individual living with learning needs must give their consent to any therapy decisions [8]. Teenagers with learning disabilities typically between the ages of 13-18 have self-harmed by cutting, and that this population is more likely to have mental health concerns [7]. It is suggested individuals' self-harm for a variety of reasons in many cases "to feel something" or to distract from emotional pain [7]. It is stated that those with an intellectual disability do self-harm more frequently when they are "emotionally dysregulated", this means they feel overwhelmed by an emotion such as anger or sadness and that their emotion has complete control [7]. It is also suggested that this group find it difficult to use effective communication or have coping skills such as talking to a friend or carer when they are feeling stressed and as a result of their emotions overwhelming them they will self-harm.

Those client's with intellectual disabilities and emotional needs must have the same access to health, education and social care as anyone else, support and adjustments are mandatory under the equality act [9].

It is acknowledged by the authors that the detriments that effect health go beyond the scope of this study which include, biological, social, political, economic, cultural, psychological, spiritual, lifestyle issues and attitudes that include negative stereotypes which may lead to discriminatory practice. Practitioners in any discipline are required to reflect on their own knowledge, attitudes and skills in working with people living with intellectual disabilities. Numerous international research studies have echoed the same message that individuals living with intellectual disabilities continue to be over prescribed medications, [7] this may be due to limited NHS resources and lack of knowledge around Integrated Therapies such as Kinetic Shift Therapy. NHS services consistently fail to work with providers in the third sector where access to holistic and integrated therapies is predominately practiced [10]. According to the world Health Organization [11], 15\% of the world's population live with a disability $4 \%$ has a significant mental disability like an intellectual disability; this is set to rise due to an increase in the global population. Who states in their mental health reports that many of the world's population do not have access to timely effective psychological therapy due to lack of training in complex areas and communication barriers which largely affect those with intellectual disabilities. The authors of this study assert the need for a more flexible, integrated/holistic approach to mental health care especially in psychological therapies when dealing with individuals with complex needs such as intellectual disabilities.

\section{Kinetic Shift Therapy}

This experimental pilot study is important due to the many challenges those with intellectual disabilities experience which includes profound issues that individuals encounter with cultural and communication issues. Kinetic Shift Therapy is one of the newer imagery-based energy psychological methods that have noticeable positive results for individuals with intellectual disabilities. Kinetic Shift Therapy was initially developed by an ex-British Army soldier and ex armed police personnel-Karl Smith [6] who trained as a clinical hypnotherapist to help people who experienced trauma after his own experience of post trauma while in the British Police Force [12]. The Kinetic Shift Protocol was taught to the authors by 2 former students of Karl Smith. One a qualified Clinical Integrated Therapist 
and EFT (Emotional Freedom Therapy) practitioner-Andy Steele [10], who is a former British Army Para trooper and ex-fire fighter in the UK who also experienced severe post trauma due to his service years, and Nicky Forbes, [13], a former experienced Complementary Therapy Lead in the NHS/Charity sector in the UK and a EFT tutor/ mentor. Steele A [10], describe KS as rapid method for resolving fears, phobias anxieties and stress, he asserts KS can assist also with reducing and eliminating behavioral issues. Forbes N [14] describes how our minds and bodies work together when we have a thought, this creates a feeling which triggers our brain into action whereby natural chemicals create the physical sensations we experience. Kinetic Shift has nearly a 1000 qualified practitioner's around the globe who come from many backgrounds-medicine, nursing, psychology, holistic and complementary therapies and hypnotherapy. The therapy mainly focuses on visualization, breathing, and sensing ability. This therapy is highly beneficial for clients who struggle with cognition, speech, and expressing emotions. Kinetic Shift holds on traditional methods of psychotherapy treatment, which do not focus on sensation and imagery, thereby needing the clients to describe their stress.

Kinetic Shift is a model based on six phases directed by a professional practitioner. The model aims at blocking arousal on pathways to the mid brain where negative memories and thoughts are kept. The body responds to the Kinetic Shift imagery by calming down through distinguishing perceived and non-existent threats. The Kinetic Shift process allows the limbic system to adapt to a calm response.

Smith asserts in his book "There is No D in PTSD" how Kinetic Shift is a way to help the individual to change their mental state and work on a deeper level with emotions and feelings [14]. The practice draws from imagery and eye movements which have both been widely researched in the past with a therapy frequently accessed in the NHS-Eye Movement Desensitization and Reprogramming (EMDR), however there remains an in-depth spoken element to the EMDR as the current protocol in the NHS draws from the Cognitive Behavioral Model-(CBT) [5], when processing distressing memories and events.

Smith states the kinetic shift approach helps if you "feel" there is a problem, but cannot express it [6]. Kinetic shift practitioners are trained to "pay attention" not to what is said by a client but to observe over any statement what the client is communicating, something which has proven unique and useful for this present study, and for those living with intellectual disabilities that struggle to express their emotions.

When working with client's who have been medically diagnosed with "Downs Syndrome" it has been found that the use of imagery improves memory and the utility of numbers, colors, shapes has resulted in a greater interest in the process from the client [9], the authors of this study advocate for this reason the application of the Kinetic Shift therapy was appropriate and beneficial for this client group. In Bhaumik et al 2011 study, they ascertain that the problem with psychotherapy techniques like Cognitive Behavioral Therapy $[\mathrm{CBT}]$, which is a mainstream therapy for all [1], is that reliance on language within this approach which limits its efficacy with client's living with intellectual disabilities.

\section{Mindfulness Therapy}

The primary focus of mindfulness-based therapy for clients with an intellectual and learning disability are shifting their concentration and allowing them to respond to triggering situations and events calmly [8]. Mindfulness can be either trained to the client or learned through self-reports using assessment questionnaires. Participants who undertake mindfulness therapy recover from self-harm behaviors such as skin picking, aggressive behavior and self-harm [15]. Kinetic Shift Practitioner and Integrated therapist Steele [10] states, that meditation practice like mindfulness therapy increases positive emotions and decreases anxiety and stress which improves the ability to regulate emotions.

Research studies have shown $[2,8,9,15]$ that regular meditation/ mindfulness practice improves the conduct and behavior of those living with a learning disability that have socially inappropriate sexual arousal issues. In this present study the client self-reports and carer reports noted a significant improvement in self-harming behavior/ aggression, and anxiety and depression following the mindfulness therapy alongside kinetic shift therapy. Mindfulness therapy, according to Harper S, et al. [15], also improves social functioning and academic performance.

Mindfulness therapy can be of benefit to people living with an intellectual disability as it is not intrusive and does not involve medication and yet improves the well-being of the clients [15]. Whittle $\mathrm{C}$ and Butler $\mathrm{C}$ in their study [11], state any practices that has a positive impact on the mental health of those living with intellectual disabilities is important to prevent isolation and a negative self-image.

Mindfulness based therapy, had traditionally been trialed in setting with those individuals who have suffered with chronic long term conditions however they appear in settings such as mental health and with learning disabilities in more recent times [16].

There still remains a lack of robust evidence to suggest the usefulness of mindfulness in decreasing behavior and social and emotional problems of young adults living with an intellectual disability [17]. It has been suggested by some studies that there has been a substantial improvement in the report symptoms of anxiety and depression and aggressive behavior when trialed [8], however it is stated that further specific tailored research is needed. Other studies have found a positive impact on hyperactivity among this population after mindfulness therapy and similar results were found in other studies $[2,18]$ which found that young adults with less than optimal emotional regulation skills did lead to a poorer prognosis in all aspects of daily life; these individuals also displayed more behavioral problems [2].

\section{Research Sample}

This pilot study was carried out with a group of 12 male clients aged 18-21 from West Yorkshire in the United Kingdom who had intellectual disabilities. The young men had self-harming behavior around their sexuality, these included-cutting arms, wrists, legs, stomach and pulling out their hair, co-existing depression, and anxiety and anger symptoms were present. The clients had previously been identified with intellectual disabilities using the DSM-5 criteria [17], the discussion around this goes beyond the scope of this study. The client's independently and willingly agreed to the study being carried out and were asked on two separate occasions by an independent person in association with a local charity for those living with learning needs. The kinetic shift and mindfulness protocols were reviewed by two psychologists prior to the test and approval given. The research aimed to establish whether the Kinetic Shift Therapy and Mindfulness Therapy would be of benefit to this population.

\section{Research Design and Method}

The study used the quasi-experiment one group pre and post-test design to gather information from the participants. Ethics approval was obtained via the Charity and followed guidelines as set out by the UK National Institute of Health Research (NIHR). Mindfulness therapy and Kinetic Shift therapy information and breathing techniques 
information was given to the clients and their carers before the study, as well as information on the practitioner in case of inquiries. The study took place in one day, whereby the participants were asked questions considering their emotional health and self-harming behavior such as infliction of cuts on limbs and stomach due to the inability to express themselves. Caregivers of the participants were given the task of helping them fill the pretest and posttest questionnaires and forms, which they did and returned after a month pre and post testing.

\section{Results}

This present study has been inspired by training courses the author's attended on Kinetic Shift Therapy and Mindfulness Based Therapy. All practitioners received Clinical Supervision of 1 hour before the group/ individual therapy commenced and 1 hour following to ensure correct procedural validity.

The clinical trial supervisor ensured all protocols were followed, 2 psychologists reviewed all information pre and post testing. Within the study assessment tools were used to evaluate symptoms and behavior in the client's with intellectual disabilities. Practitioner's and the clinical supervisor were around to explain the scoring system for the client's and their carers. The self-report and carer report included items which asked both parties to rate how they agreed with statements such as (1=never to $4=$ always). The measurements showed a high test (and a high rest-test reliability) $(\mathrm{r}=6.7)$ which also led to a consistency of $(a=0.7-0.8)$ which was high and enhanced the validity clinically. A variety of sub scales were looked at such as self-harm, aggression, anxiety and depression.

Data Analysis-the main hypotheses were tested with ANOVA. A natural logarithm was used as a formula. Power analysis of the statistics had been implemented using software (GPOWER $\left.{ }^{\star}\right)$

Demographically the data involved the use of descriptive statistics whereby a natural logarithm in a formula was utilized where all scores were transferred.

\section{Process}

Clients were taken through a four-hour session of therapy with a short break, whereby they first joined a support group. The clients were taken individually for private Kinetic Shift therapy in the first hour, which included the use of imagery-based interventions and the recordings of subjective units of distress (suds). 3 trained Kinetic Shift Practitioner's and 1 Mindfulness based interventions practitioner took part in the study to guide the participants.

A relaxation/body scan was carried out where emotions were identified and areas where they were felt in the body. Face-to-face talks were carried out discussing thoughts, feelings and behavior in the format of colors, shapes, objects and numbers. The session ended with mindfulness therapy and recordings which were given to the clients and key workers to take home and go through. The pretests questionnaire was completed on the morning before the therapies and the posttests 1 month after.

The feedback collected from the assessment forms returned by the clients proved that the hypothesis of this study is correct. The questionnaires showed improvement in how the participants regulated their emotions, and they were able to release painful emotions, which reduced self-injury quickly. Anxiety, depression, and self-harm levels had reduced after the therapy was conducted. This study, therefore, suggests that Kinetic Shift Therapy and Mindfulness Therapy is beneficial and yield positive results to clients living with an intellectual disability.
12 young men took part in the study the mean age was 18.5, the information from the statistics did correlate with the client's selfreport of depression symptoms which was negative $(r=-58, p$ is more than 0.02) carers reported inattention $(r=-52$, more than 0.01$)$, the clients were more likely to report-self harm and anxiety, and the carers reported conduct, aggression, and self-harm type symptoms.

Within the client's self-report questionnaires. Bonferronia was used (bonferronia correction) this was to compare post hoc-the score of the anxiety scales were noted at pre-tests that these were higher than the scores at post tests for anxiety ( the mean), this was also applicable to all scores/the mean scores. Using Wilkes which was determined at +.66 - (f), and $[17,19]$ did equal 6.72 , it was determined at $0.02=p$, which was equal to 6.73 , plus the mean depression score at pre-test was also higher from the mean score at post test.

The carer report questionnaires were conducted with the same procedure and highlighted the benefit of Kinetic Shift and the mindfulness based protocols with the client who expressed internal and external symptoms. The results from the carer reports revealed significant differences in how they perceived their client's external symptoms, it could be noted that there was difference in the scoring and noted that Wilkes increased $=0.50$, which was $\mathrm{f}=(1,12)$ which was equal to the score 8.26, and 0.2 did equal p, n3 $=0.40[19,20]$. Again comparisons presented that the meant that the score for symptoms of aggression at pre-test was on the high side, from the scores at posttest, this was noted with self-harm and other problems identified in the trial. It can be ascertained that there were significant variances in how the client's perceived their internal mental health symptoms to how their carers perceived them as shown on (Figure 1 and Figure 2).

Analysis of sensitivity was used to describe the impact of any additional violation/outliers of distributions through transforming the logarithms. The Friedman test was utilized for this. Our analysis of the data determined that the results were robust with the chosen protocols.

Analysis of the sub scales looked at where our Kinetic Shift/ Mindfulness interventions had the greatest effect, there were many changes pre to post test in the client's responses such as when answering questions like "I am afraid that I will do something bad to myself "was reported by $29 \%$ of the client's in their self-report as was "I get so anxious I cannot breathe." In the aggression sub scale those areas most improved was "he defies his carers" and "he teases other residents in the home" these external symptoms/behaviors were improved by the Kinetic Shift/Mindfulness intervention.

\section{Strengths/Limitations/Conclusion}

This study has much strength, for example, the clients were very specific with moderate learning disabilities which presented a uniform group of people with regards to their mental health diagnosis. Attrition did not appear present in this pilot study which strengthened the above results, and the analysis of the sensitivity did strengthen our final conclusions (Table 1).

This study notably contributed to the growing body of evidence that already exists for those who live with intellectual disabilities, plus the inclusion of kinetic shift as an intervention which has proven to help those with external and internal emotions and behavior conflicts, appears critical when discussing treatment protocols for a group of individuals who struggle with learning/communicating and understanding their feelings/emotions and behavior. Kinetic Shift practice also adds to the body of research which is available in areas such as energy psychology. Finally, this is the only pilot study that 


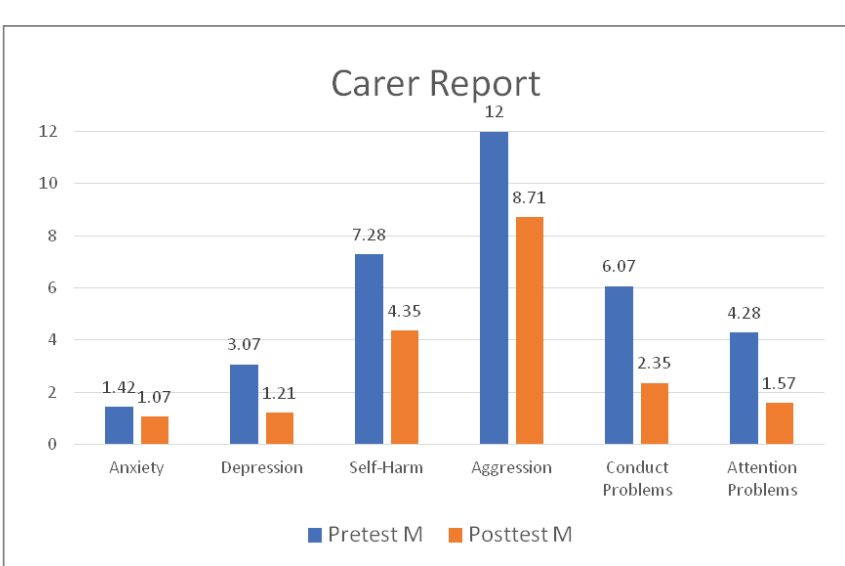

Figure 1: Carer Report.

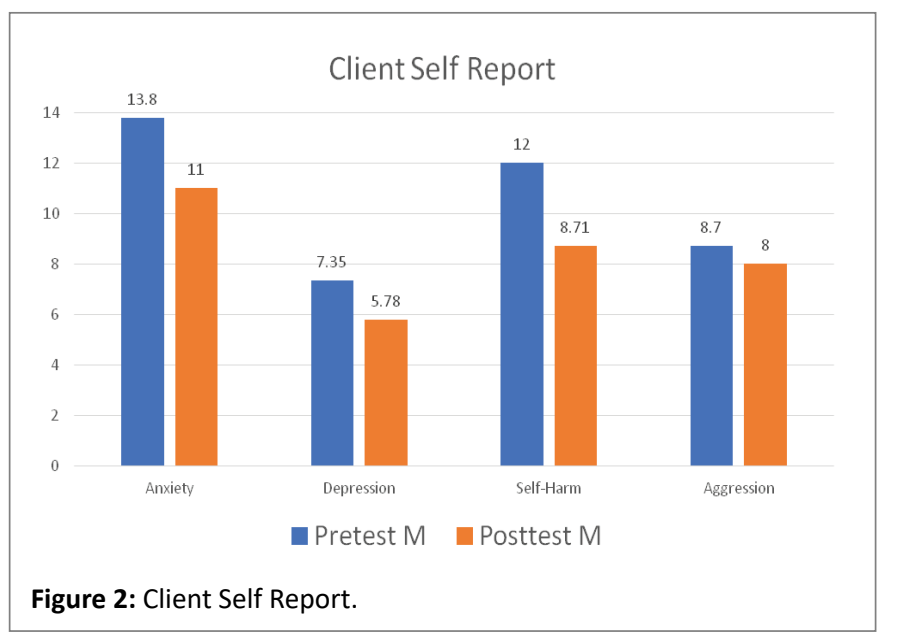

evaluated kinetic shift alongside mindfulness to help those living with Intellectual Disabilities with enduring mental health problems.

One limitation was that the 1 group pre-tests-post-tests design was unique without a control group. The changes that were noted and observed did not identify if the changes and positive effects of the treatment was due to kinetic shift, mindfulness or either or neither. Another limitation was that the sample size was small, and the assessment that took place were limited to pre and post testing in a limited time span, however due to robust testing and analysis this did create a validity. The analysis of the sensitivity suggests that the power statistically was robust. Some sub scales were affected by the pre/pest test method however which was again due to the 1 group method.

It is also not clear as such to determine if the forms/assessments undertaken by the carer's of the client's in the trial had any bias with regards to any positive changes in the 1 group and pre/post testing method. Another limitation is that lack of follow up as this was specified to one-month post-test a longer time frame is required to assess the full benefits of the Kinetic Shift/Mindfulness intervention.

The conclusions from this research trial are promising; this scientific study does suggest the positive effects of both Kinetic Shift Therapy and Mindfulness Based Therapy for this client group. The use of a randomized controlled trial is strongly recommended as is testing Kinetic Shift therapy in its own right with this population.
Table 1: Repeated measures ANOVA for $\mathrm{C}$ and AMM/BAS-1/Means, Standard Deviations.

\begin{tabular}{|l|c|c|c|c|}
\hline \multicolumn{1}{|c|}{$\begin{array}{c}\text { Client } \\
\text { assessment }\end{array}$} & Pretest M & Pretest (SD) & Posttest M & Posttest, M (SD) \\
\hline Anxiety & 13.8 & -7 & 11 & $11.00(6.60)$ \\
\hline Depression & 7.35 & -4.4 & 5.78 & $5.78(5.30)$ \\
\hline Self-Harm & 12 & -5.7 & 8.71 & $8.71(4.10)$ \\
\hline Aggression & 8.7 & -4 & 8 & $8.00(4.20)$ \\
\hline $\begin{array}{l}\text { Carer } \\
\text { assessment }\end{array}$ & Pretest M & Pretest (SD) & Posttest M & Posttest, M (SD) \\
\hline Anxiety & 1.42 & -1.4 & 1.07 & $1.07(1.60)$ \\
\hline Depression & 3.07 & -3.8 & 1.21 & $1.21(1.80)$ \\
\hline Self-Harm & 7.28 & -6.8 & 4.35 & $4.35(3.00)$ \\
\hline Aggression & 12 & -5.7 & 8.71 & $8.71(4.10)$ \\
\hline $\begin{array}{l}\text { Conduct } \\
\text { Problems }\end{array}$ & 6.07 & -6.5 & 2.35 & $2.35(1.90)$ \\
\hline $\begin{array}{l}\text { Attention } \\
\text { Problems }\end{array}$ & 4.28 & -4.5 & 1.57 & $1.57(1.50)$ \\
\hline
\end{tabular}

Abbreviations: AMM and (child): Adolescent Mindfulness Assessment/ Measures (and child); BAS: Behavioral Assessment System; ANOVA: Variance Analysis

* $\mathrm{P}=$ less than 0.5

$++P=$ less than 0.1

$\mathrm{M}=$ Mean

$\mathrm{SD}=$ Standard Deviation

Kinetic Shift alongside Mindfulness therapy can be described from the results as a method that will make it possible to help relieve and prevent the mental health symptoms in clients with enduring mental health problems with a moderate learning disability. This pilot study has shown how it has been possible to help decrease unpleasant emotions and increase tolerance to anxiety/depression and selfharming thoughts and symptoms.

\section{References}

1. Bhaumik S, Watson J, Barrett M, Raju B, Burton T, et al. (2011) Transition for Teenagers with Intellectual Disability: Carers' Perspectives. Journal of Policy and Practice in Intellectual Disabilities 8: 53-61.

2. Carmen S (2017) Persons with multiple disabilities. The British Journal of development and disabilities 67 .

3. Smith K (2020) Karl Smith hypnotherapy.

4. NICE (2018) Post-traumatic stress disorder. National Institute for Health Care and Excellence, UK.

5. United Kingdom Hypnosis Academy UKHA (2019).

6. Stoffelen C (2018) Maastricht University.

7. Rushbrooke E, Murray C, Townsend S (2014) The Experiences of Intimate Relationships by People with Intellectual Disabilities: A Qualitative Study. J Appl Res Intellect Disabil 27: 531-541.

8. Griffith G, Hastings R (2019) Good Practice Guidelines: Mindfulnessbased programs for people with Learning Disabilities.

9. Mencap (2020).

10. Steele A (2020) Andy steele phoenixtherapies. 
11. Whittle C, Butler C (2018) Sexuality in the lives of people with intellectual disabilities: A meta-ethnographic synthesis of qualitative studies. Res Dev Disabil 75: 68-81.

12. NICE (2018) Post-traumatic stress disorder overview.

13. Forbes N (2020) Nicky Forbes therapies.

14. Smith K (2017) There is No ' $d$ ' in Ptsd. Kindle edition, Amazon Digital Services LLC-KDP Print US.

15. Harper S, Webb TL, Rayner K (2013) The Effectiveness of MindfulnessBased Interventions for Supporting People with Intellectual Disabilities: A Narrative Review. Behav Modif 37: 431-453.

16. RCN (2019) Mental Health Act Review.
17. RCN (2019) Intellectual disability protocols.

18. American Psychiatric Association (2013) Diagnostic and Statistical Manual of Mental Disorders. $5^{\text {th }}$ Edition. American Psychiatric Publishing, USA.

19. Ougrin d, Boege I (2013) Brief report: The self harm questionnaire: A new tool designed to improve identification of self harm in adolescents. Journal of Adolescence 36: 221-225.

20. Achenbach TM (1991) Manual for the Child behavior checklist/4-18 and 1991 profile. Department of psychiatry, Burlington VT: University of Vermont. 